\title{
Hypertrophic cardiomyopathy and hyperthyroidism: a report of three cases
}

\author{
C. S Y MONS,P.J.RICHAR D S N, \\ a n d O. F E I Z I \\ Department of Cardiology, Royal Free Hospital, London NW3 2QG
}

\begin{abstract}
Symons, C., Richardson, P. J., and Feizi, O. (1974). Thorax, 29, 713-719. Hypertrophic cardiomyopathy and hyperthyroidism. The combination of hypertrophic cardiomyopathy and hyperthyroidism gives rise to a complex clinical picture as some of the symptoms and signs may be common to both conditions. The presentation and investigation of three patients are reported. In one patient there was evidence of progression from the hypertrophic obstructive phase to that associated with loss of outflow tract obstruction. The echocardiogram was especially useful in assessing the presence or absence of hypertrophic disease in the thyrotoxic subject. It is suggested that the long continued high-output circulatory state in clinically undetected hyperthyroidism may prove to be a stimulus for unrestrained cardiac muscle hypertrophy.
\end{abstract}

Three patients are described, each of whom, at one time, had evidence of both hypertrophic cardiomyopathy and hyperthyroidism. Although the association of the two conditions may be fortuitous, the combination has not previously been reported, and the symptoms and signs pose problems in diagnosis which merit discussion. In addition, the extra work-load inherent in the maintenance of the hyperkinetic circulation in thyrotoxicosis might have some bearing on the aetiology of hypertrophic cardiomyopathy. Abnormal echocardiograms were recorded in each, and this technique proved a useful and simple method of screening these thyrotoxic patients when cardiomyopathy was suspected and prior to more detailed investigation by cardiac catheterization and angiography.

\section{CASE REPORTS}

PATIENT 1. A 38-year-old woman was referred to the cardiac clinic in 1958 because of a murmur. This had been noted by the general practitioner in 1956 but had not previously been commented upon at earlier routine examinations. Clinically an anxious woman - of average build, the only physical abnormality was a grade 3/4 mid-to-late apical systolic murmur which ceased before the second sound. The blood pressure was $130 / 80 \mathrm{mmHg}$. The chest radiograph was normal. The electrocardiogram revealed sinus rhythm, frontal plane QRS axis $-15^{\circ}$, a small $Q$ in aVF, PR interval 0.12 sec. In 1959 she had attacks of paroxysmal supra- ventricular tachycardia, and a year later chest pain and shortness of breath on exertion occurred. In 1961 the electrocardiogram showed a pre-excitation type B pattern, PR interval $0.08 \mathrm{sec}$, frontal plane QRS axis $+10^{\circ}$ (repeated records remained almost identical henceforward). There was no alteration in the physical signs in the cardiovascular system but hyperthyroidism was suspected because of undue anxiety, tachycardia, and a small midline thyroid nodule. However, relevant investigations were negative (BMR 9\%, ${ }^{131}$ I thyroid uptake $25 \%$ at 4 hours and $50 \%$ at 48 hours).

The patient was followed up routinely and in 1968 typical thyrotoxicosis developed, thyroid enlargement becoming more obvious, although there were no eye signs. The diagnosis was confirmed with thyroid function studies (protein-bound iodine 9.5 and $11.2 \mu \mathrm{g} /$ $100 \mathrm{ml},{ }^{131}$ I thyroid uptake $68 \%$ at 4 hours). A subtotal thyroidectomy was performed and histology showed a multinodular goitre with areas of epithelial hyperplasia and absorption of colloid.

The patient improved but the following year developed palpitations and attacks of paroxysmal nocturnal dyspnoea, necessitating full cardiac investigation. The chest radiograph revealed left ventricular enlargement, the electrocardiogram was unaltered, and an echocardiogram was abnormal (Fig. 1), showing a prominent forward systolic displacement of the anterior mitral valve leaflet together with a reduced diastolic closure rate and evidence of a thickened septum. At cardiac catheterization the left ventricular pressure was $240 / 20 \mathrm{mmHg}$ and there was an intraventricular systolic gradient of $140 \mathrm{mmHg}$; there was 


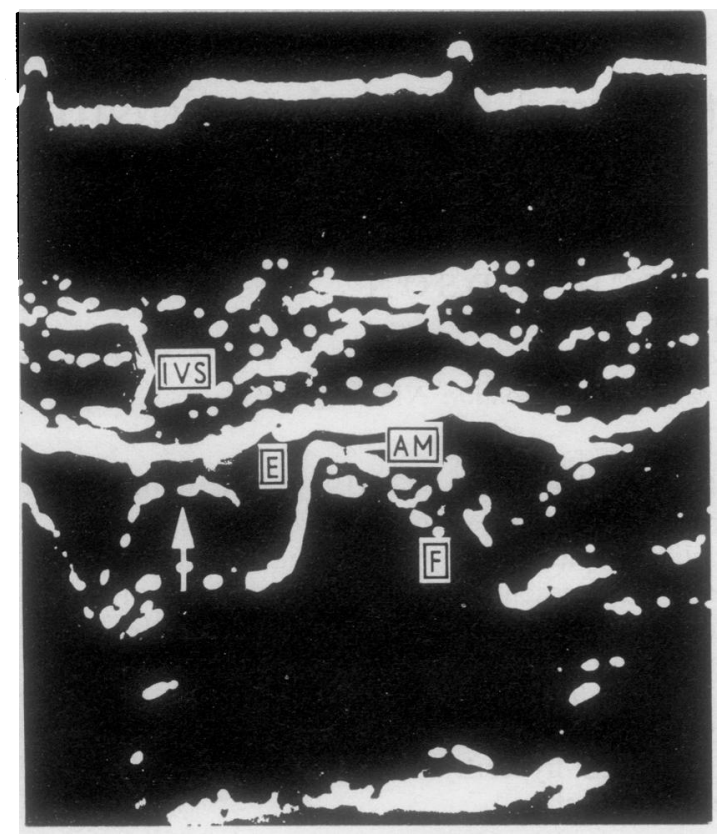

FIG. 1. PATIENT 1. Echocardiogram showing abnormal systolic forward displacement (arrowed) of the anterior mitral valve leaflet $(A M)$, prominent interventricular septal echoes (IVS), and closure of mitral valve during mid-diastole $(E, F)$.

no systolic gradient across the aortic valve. A left ventricular angiocardiogram showed obliteration of the distal part of the left ventricular cavity with severe septal hypertrophy and mitral incompetence.

Despite medical treatment the patient deteriorated, cardiac surgery was considered, and eventually a resection of muscle from the left ventricular outflow tract was performed. At operation there was marked thickening of the left ventricular wall with additional hypertrophy of the whole of the septum as far as the aortic valve. There was no localized stenosis and no abnormality of the mitral valve. The patient died a few hours after operation. A myocardial muscle biopsy taken at operation revealed classical changes of hypertrophic obstructive cardiomyopathy (Fig. 2). Necropsy showed that there was no coronary artery disease, no intrinsic mitral leaflet abnormality, and no cardiac infarct. The small coronary vessels were normal; no microscopic examination of the conducting tissue was made.

PATIENT 2. In March 1969 a woman aged 53 was admitted with acute left ventricular failure considered to be due to non-rheumatic mitral incompetence. There was no previous relevant history of cardiac disease; pernicious anaemia had been diagnosed in 1956, for which regular B12 injections were given. The protein-bound iodine was raised, $8.9 \mu \mathrm{g} / 100 \mathrm{ml}$, but no further investigations were carried out. In음 August 1969 she complained of weight loss, anxiety,으 orthopnoea, and palpitation and expressed a pre- $\overline{\bar{C}}$ ference for cold weather. Examination revealed a thin $\vec{\nabla}$ anxious woman with a palpable thyroid gland, hand $\varrho$ tremor, and palmar erythema; tachycardia wasw present. There were no eye signs. The pulse was of $\overrightarrow{0}$ full volume with a rapid upstroke, the blood pressure-was $120 / 80 \mathrm{mmHg}$, there was evidence of left ventri- $\vec{\omega}$ cular hypertrophy, and a grade $3 / 4$ late apical systolico murmur was audible. The chest radiograph was normal but the electrocardiogram showed evidence of moderate left ventricular hypertrophy, frontal plane. QRS axis of $0^{\circ}$. Thyroid function tests confirmed? thyrotoxicosis (protein-bound iodine $9.6 \mu \mathrm{g} / 100 \mathrm{ml}, \overrightarrow{\mathrm{C}}$ T3 resin uptake $121 \%$, thyroxine iodine raised, $\omega$ ${ }^{131}$ I thyroid uptake $56 \%$ at 4 hours and $54 \%$ at 48 hours).

The patient improved with carbimazole but as the murmur remained loud detailed cardiac investigation was carried out. The echocardiogram (Fig. 3) showeds a small forward systolic displacement of the anterior mitral valve leaflet with a normal diastolic closure rate. There was evidence of gross septal hypertrophy. $\vec{c}$ At catheterangiography the left ventricular pressure ${ }_{\perp}$ was $160 / 14 \mathrm{mmHg}$, there was septal and papillary muscle hypertrophy, and a moderate degree of mitrab regurgitation completed the picture of hypertrophic cardiomyopathy (Fig. 4). When the patient becames euthyroid repeat angiography and echocardiography showed no changes in the features of hypertrophic cardiomyopathy, although the electrocardiogram had become normal.

The patient had improved after 18 months of medical treatment but when this was stopped relapse occurred and radio-iodine was administered. To date. her general condition is good, although the murmur is still loud and the systolic forward displacement of the mitral valve is still evident in the echocardiogram.

PATIENT 3. In 1956, thyrotoxicosis was diagnosed in a 32-year-old woman who was treated with carbio mazole and thyroid extract for four years. An electro $₹$ cardiogram had shown occasional sino-atrial blocko and established second-degree atrioventricular block with Wenckebach phenomenon. The chest radiograplo showed cardiac enlargement. She responded well to the antithyroid treatment, but in 1966, while inf Holland, a saddle embolism occurred during an epi sode of atrial fibrillation, and bilateral femoral arteriap embolectomy was performed. Postoperatively the. patient had a thyrotoxic crisis. Treatment with carD్ర bimazole was re-instituted and improvement was rapide It was thought that an apical mid-to-late systolic mur $\frac{f}{\Phi}$ mur was due to rheumatic mitral disease but in retros spect the accompanying carotid arterial trace (Fig. 5) appeared identical with that frequently recorded in 5 hypertrophic obstructive cardiomyopathy. The patient品 was first seen at the Royal Free Hospital in 1968 when? her general condition indicated an euthyroid state with exophthalmos. No cardiac murmurs or abnormab 


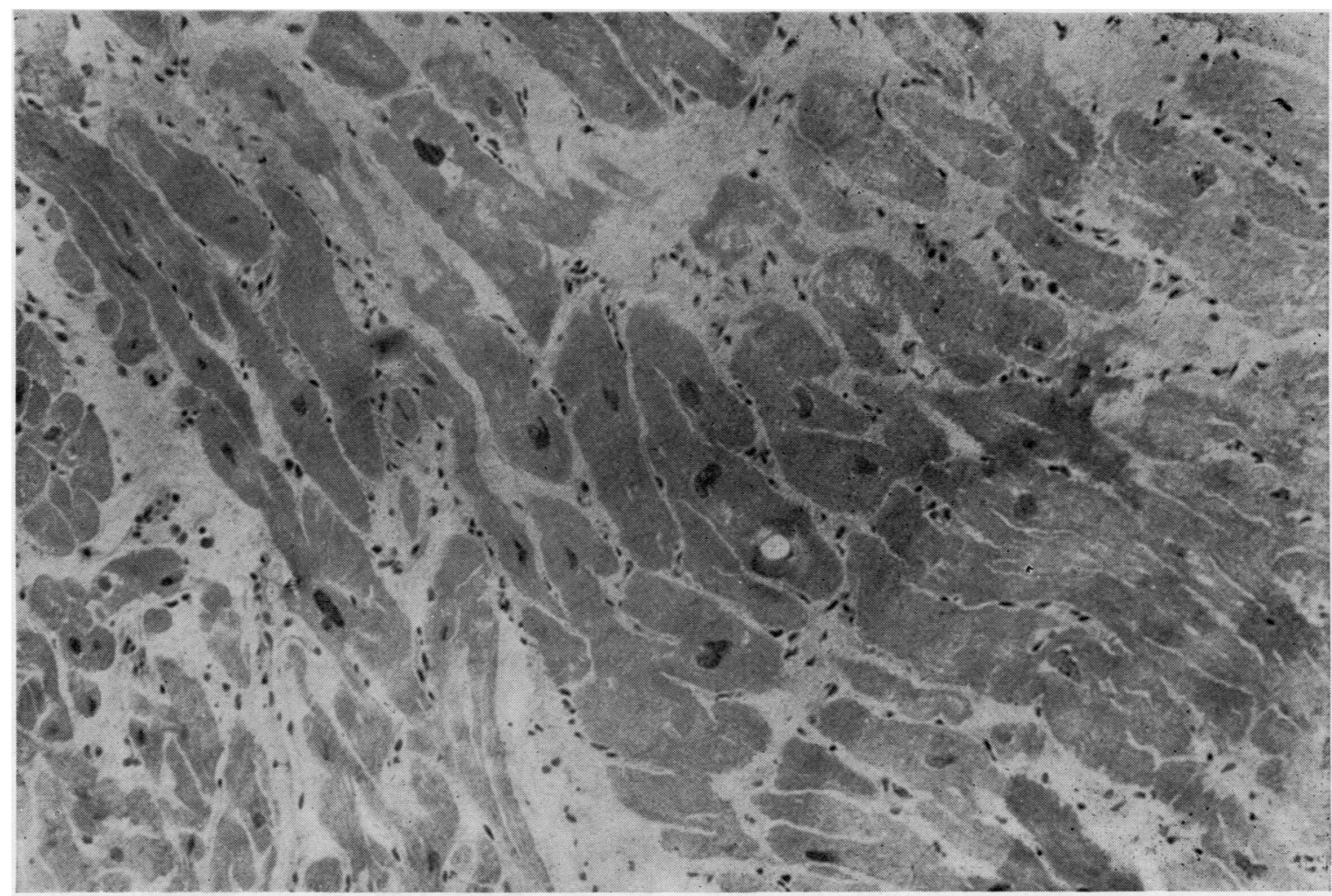

FIG. 2. PATIENT 1. Left ventricular muscle showing characteristic changes of hypertrophic cardiomyopathy, viz, directional disorganization of muscle fibres, increase of connective tissue, and 'short appearing' fibres. $H$ and $E$ cryostat section $\times 120$.

sounds were audible or recorded phonocardiographically. Atrial fibrillation was present, blood pressure was $130 / 80 \mathrm{mmHg}$, and the electrocardiogram showed evidence of moderate left ventricular hypertrophy. Carbimazole treatment was stopped but the following year thyrotoxicosis recurred. Radio-iodine therapy was given and the thyroid condition again improved, but in 1969 increasing dyspnoea, especially nocturnally, and an abnormal echocardiogram suggested the diagnosis of cardiomyopathy. The echocardiogram (Fig. 6) showed a fast diastolic closure rate of the anterior mitral valve leaflet with a secondary re-opening during mid-diastole; movement of the posterior ventricular wall and septum was reduced. No forward systolic displacement of the mitral valve was evident. Right heart catheterization revealed an indirect mean left atrial pressure of $24 \mathrm{mmHg}$, rising to $32 \mathrm{mmHg}$ after exercise. The pulmonary artery pressure was $53 / 20$ with a mean of $36 \mathrm{mmHg}$. The left heart was not catheterized. In view of the deterioration in the patient's condition exploratory cardiac surgery was carried out. No valvar disease was present but limited examination by palpation through the mitral valve revealed marked thickening of the left ventricular muscle with increased cavity size. A biopsy was taken near the cardiac apex, and this showed gross hyperplasia of the muscle fibres with marked dystrophic changes. There was a considerable degree of fibrosis and elastosis, and some increase in glycogen in the perinuclear areas (Fig. 7). Two days after operation the patient developed a right hemiplegia, presumably due to thromboembolism. Convalescence was slow. Carbimazole was continued and there have been no further episodes of left ventricular failure (possibly related to the marked restriction of physical activity). Currently the physical findings, the electrocardiogram, and the echocardiogram are unchanged.

\section{DISCUSSION}

In the three cases described the exact relationship of thyroid overactivity to the time of onset of hypertrophic disease is difficult to assess. Certainly two patients had thyrotoxicosis, one at the same time as the cardiomyopathy was present (patient 


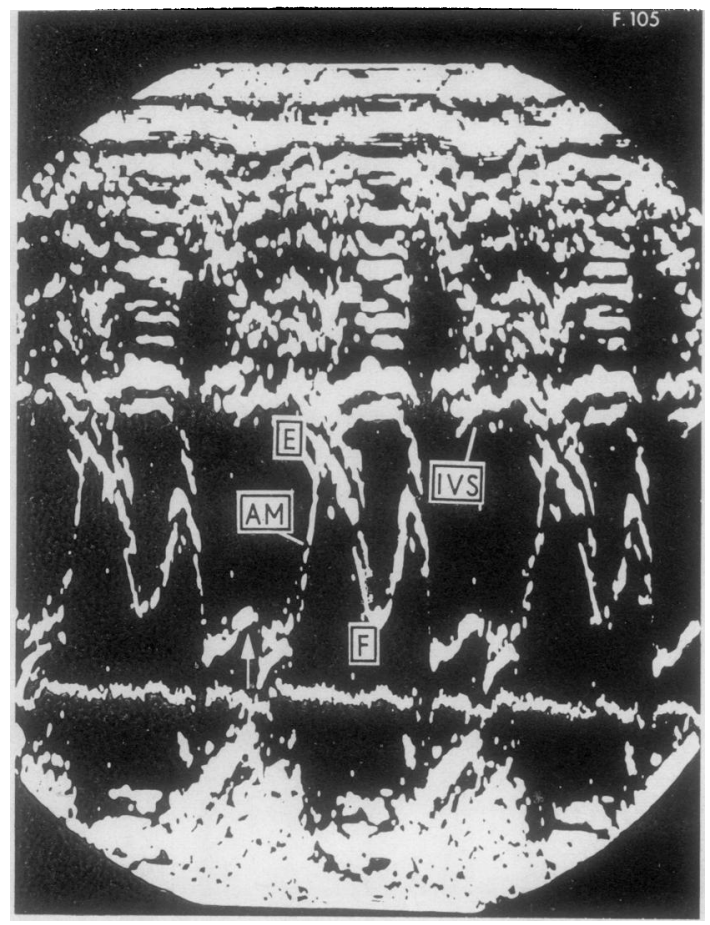

FIG. 3. PATIENT 2. Mitral valve echocardiogram showing a small forward displacement during systole (arrowed) of the anterior valve leaflet $(A M)$, interventricular septum (IVS), and closure of the mitral valve during mid-diastole $(E, F)$.

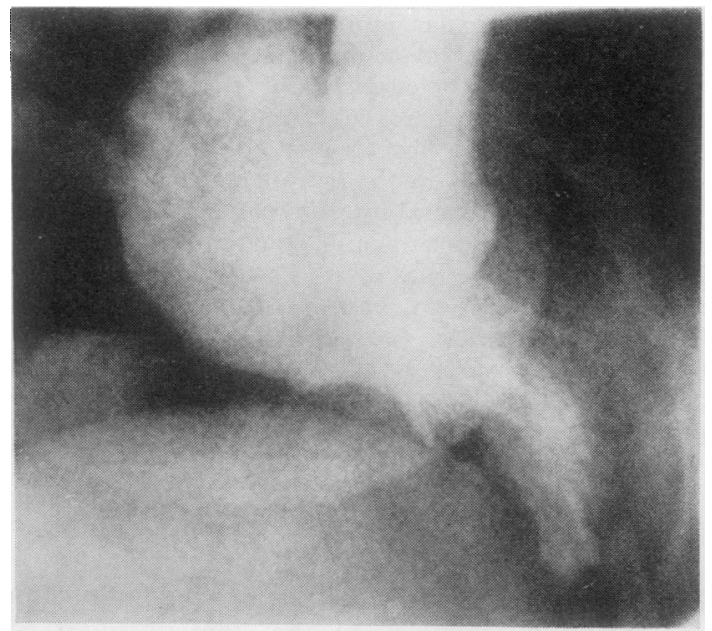

Fig. 4. Patient 2. Single frame from left ventricular cine-angiogram (right anterior oblique) showing typical features of hypertrophic cardiomyopathy (see text).

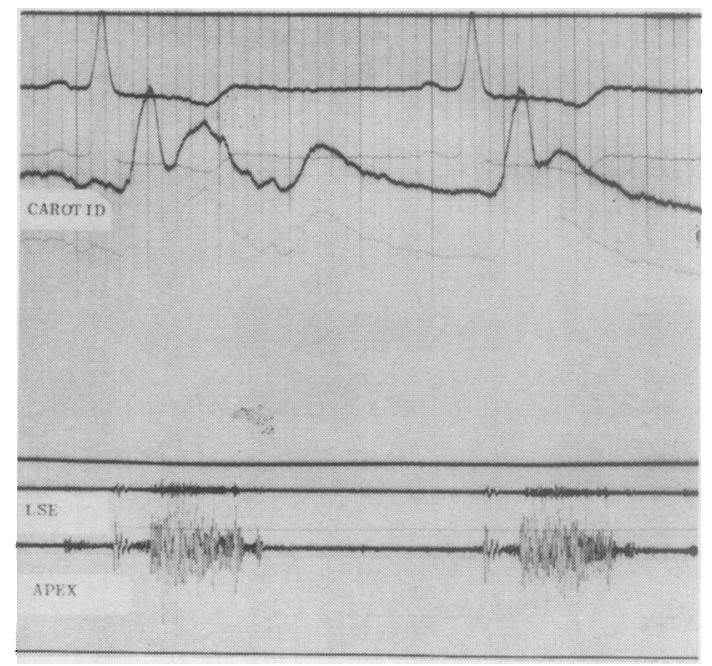

Fig. 5. PATIENT 3. External carotid pulse tracing showing rapid upstroke, sudden collapse, and sharp tidal wave. There is a mid-to-late systolic murmur.

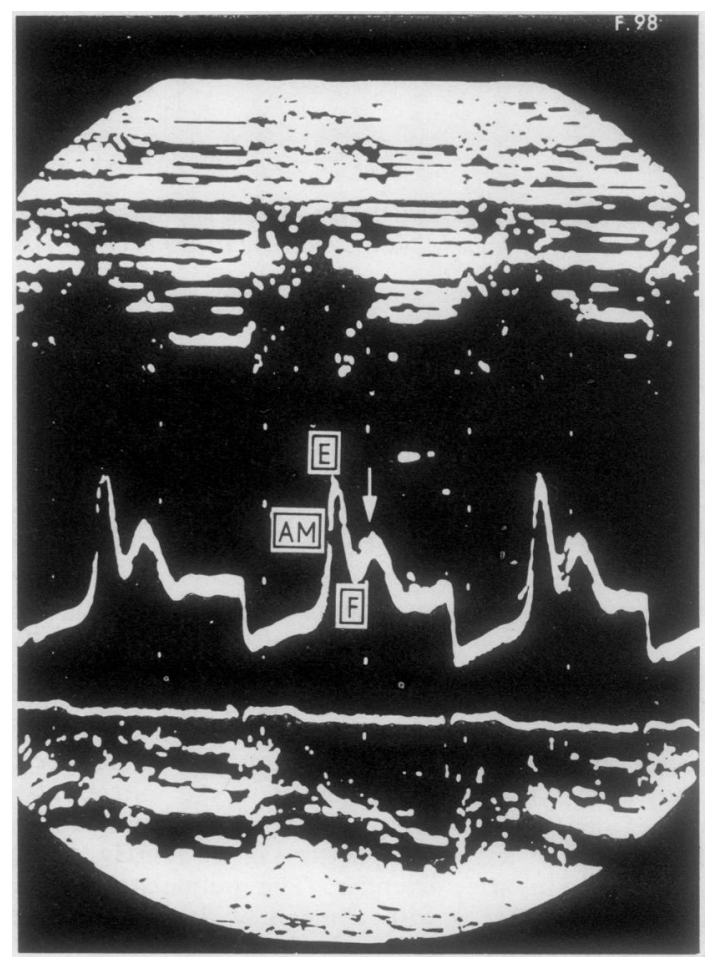

FIG. 6. PATIENT 3. Echocardiogram of mitral valve indicating a rapid diastolic closure rate $(E, F)$ and the secondary diastolic re-opening (arrowed) of the anterior mitral valve leaflet $(A M)$. 


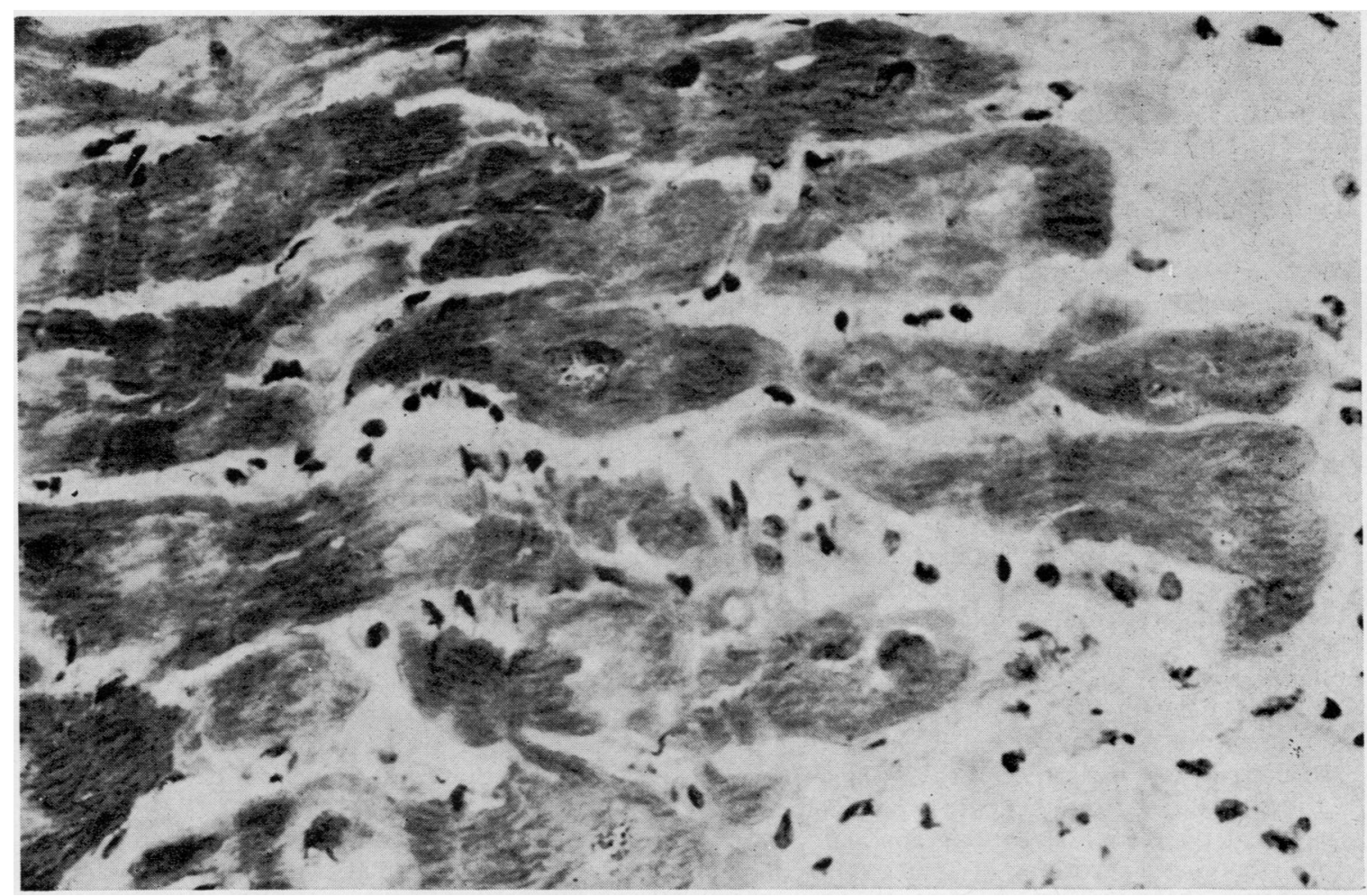

FIG. 7. PATIENT 3. Left ventricular muscle biopsy showing gross hyperplasia of muscle fibres (up to $60 \mu$ diameter) with increase of connective tissue. $H$ and $E$ cryostat section $\times 300$.

2) and the other preceding the diagnosis of cardiac disease (patient 3). However, in patient 1 a cardiac murmur had been audible for many years before the definitive diagnosis of hypertrophic cardiomyopathy was made, and during the time this patient attended the cardiac clinic underlying thyrotoxicosis had been suspected as a cause of the cardiac lesion eight years before hyperthyroidism was actually confirmed.

It is possible that each might have suffered from occult hyperthyroidism for a prolonged length of time prior to obvious heart disease, as it is known that thyroid overactivity may not be manifest clinically, particularly in older subjects, until its presentation as a cardiac disorder (Symons, Richardson, and Wood, 1971). Self-limiting periods of thyrotoxicosis have also been observed (McLarty, Alexander, Harden, and Robertson, 1971) and a repeated search for this condition may well prove rewarding in patients with hypertrophic cardiomyopathy when thyroid function is initially normal.

The clinical diagnosis of hypertrophic cardio- myopathy in the presence of hyperthyroidism is difficult to make since many of the physical signs are common to both diseases, viz, rapid upstroke pulse, mild hypertension, left ventricular type of cardiac impulse, and a systolic murmur at the left sternal edge. The murmur differs in timing in the two conditions and may be a useful diagnostic point of differentiation; in thyrotoxicosis the typical murmur is in early systole (Ueda et al., 1963) while in hypertrophic cardiomyopathy the murmur occurs later in systole (Goodwin, 1970). Although in one of our three cases no murmur or abnormal sound was either heard or recorded from 1968 onwards, it would seem reasonable to suspect the presence of hypertrophic cardiomyopathy in addition to hyperthyroidism, should a mid or late systolic murmur persist after the euthyroid state is attained. Dysrhythmias commonly occur in both conditions, e.g., multiple ventricular ecoptic beats and atrial fibrillation, and although pre-excitation has been recorded in thyrotoxicosis (Sanghvi and Banerjee, 1961), it is not possible to state if either hypertrophic car- 
diomyopathy or thyrotoxicosis was the cause of this abnormality in our first patient.

We have found echocardiography especially informative in screening patients with thyrotoxicosis as it is frequently impossible or undesirable to perform invasive investigations in these highly anxious subjects if hypertrophic myocardial disease is suspected. The characteristic abnormal forward displacement of the anterior mitral valve leaflet (Pridie and Turnbull, 1968; Shah, Gramiak, and Kramer, 1969) was demonstrated in patients 1 and 2 where there was also evidence of septal hypertrophy. The former also had a reduced diastolic closure rate of the mitral valve. The abnormalities in the third patient were different. The diastolic closure rate of the anterior mitral valve leaflet was fast but, in addition, there was secondary reopening in mid-diastole. One explanation for this would be a rapid reversal of the pressure gradient between the left atrium and left ventricle because of the high left atrial pressure and a poorly compliant left ventricular muscle. In this patient there was also a notable absence of a systolic opening movement of the mitral valve, suggesting progression in the natural history of hypertrophic cardiomyopathy, from the truly hypertrophic state suggested by the phonocardiogram and indirect carotid pressure recording of 1966 to the current clinical condition with some of the features of the phase described by Goodwin (1970) where there is a spontaneous loss of outflow tract obstruction from impairment of systolic contractile function.

Thyrotoxicosis has been known to cause cardiac hypertrophy as shown by necropsy studies, but no contemporary data exist to compare these changes with the features of hypertrophic disease as described by Goodwin (1970) and Goodwin and Oakley (1972). A search of the literature for detailed and unequivocal descriptions of necropsy findings in patients who have died after and with long-standing thyrotoxicosis indicates that impressive and abnormal left ventricular hypertrophy may occur, in keeping with the modern concept of hypertrophic myocardial disease. Symmers (1918) described six such patients, one of whom he stated had papillary muscle hypertrophy (and shown by photograph), and Loos (1929) also reported a similar case with gross left ventricular hypertrophy and a cartilage-like thickening of the papillary muscles.

The cardiac muscle biopsies which we have obtained showed the typical features of hypertrophic cardiomyopathy (Olsen, 1973) on light microscopy, histochemistry, and electron microscopy in one instance. In patient 3 the changes were not specific for this condition, showing evidence only of muscle hypertrophy and fibrosis, although the area examined was not from the most favourable site. Muscle hypertrophy could result directly from the effects of increased levels of circulating thyroid hormones and their analogues (Symons, Olsen, and Hawkey, 1974), also from the secondary effect of tachycardia and augmented cardiac output. The long-continued high output state, conceivably clinically undetected for many years, may prove to be a stimulus for unrestrained muscle hypertrophy, if some underlying myocardial fault is present. A generalized muscle disorder may be present in both hyperthyroidism and hypertrophic cardiomyopathy, for it is of interest that the electromyogram shows diffuse abnormalities in the former condition, and changes have also been recorded in voluntary muscle in subjects with hypertrophic cardiomyopathy (Meerschwam, 1969). We do not believe there is enough evidence to postulate an autoimmune basis for the myocardial abnormalities. Thyroid antibodies were negative in our patients, and in two cases where this test was performed we have not found any cardiac muscle antibodies.

Both hyperthyroidism and hypertrophic cardiomyopathy have a familial association, but none of the three patients we have described had relatives who had any unusual forms of cardiac disorder.

We are grateful to Mr. Lance Bromley, F.R.C.S., Dr. Simon Rees, Professor Everson Pearse, and Miss Susan van Noorden for their help with these cases.

\section{REFERENCES}

Goodwin, J. F. (1970). Congestive and hypertrophic cardiomyopathies. Lancet, 1, 731. and Oakley, C. M. (1972). The cardiomyopathies. British Heart Journal, 34, 545.

Loos, F. (1929). Kasuistischer Beitrag zur Frage der Herzveränderungen bei Morbus Basedowii. Zeit- $\frac{D}{O}$ schrift für Kreislaufforschung, 21, 641.

McLarty, D. G., Alexander, W. D., Harden, R. McG., N and Robertson, J. W. K. (1971). Self-limiting episodes of recurrent thyrotoxicosis. Lancet, 1, 6. N

Meerschwam, I. S. (1969). Hypertrophic Obstructive Cardiomyopathy, a Clinical Study, p. 134. Ex-

Olsen, E. G. J. (1973). The Pathology of the Heart. Intercontinental Medical Book Corporation, New York.

Pridie, R. B. and Turnbull, T. A. (1968). The aeti- $\frac{T}{0}$ ology of mitral valve dysfunction as revealed by $\frac{O}{\mathbb{D}}$ ultrasonics. In Proceedings of the 5th European Congress of Cardiology, Athens, September $\mathbb{D}$ 1968, pp. 263-272. 
Sanghvi, L. M. and Banerjee, K. (1961). WolffParkinson-White syndrome associated with thyrotoxicosis. American Journal of Cardiology, 8, 431.

Shah, P. M., Gramiak, R., and Kramer, D. H. (1969). Ultrasound localization of left ventricular outflow obstruction in hypertrophic obstructive cardiomyopathy. Circulation, 40, 3.

Symmers, D. (1918). The relationship of the so-called idiopathic cardiopathy to exophthalmic goitre. Archives of Internal Medicine, 21, 337.

Symons, C., Olsen, E. G. J., and Hawkey, C. M. (1974). Production of cardiac hypertrophy by triiodothyroacetic acid (triac). Submitted for publication.
Richardson, P. J., and Wood, J. B. (1971). Unusual presentation of thyrocardiac disease. Lancet, 2, 1163.

Ueda, H., Uozumi, Z., Watanabe, H. Kobayashi, T., Kawai, N., Matuura, T., and Iwase, T. (1963). Phonocardiographic study of hyperthyroidism. Japanese Heart Journal, 4, 509.

Requests for reprints to: Dr. C. Symons, Department of Cardiology, Royal Free Hospital, Pond Street, London NW3 2QG. 